\title{
Imprecise structural reliability analysis using PC-Kriging
}

\author{
R. Schöbi \& B. Sudret \\ Chair of Risk, Safety and Uncertainty Quantification \\ ETH Zurich, Zurich, Switzerland
}

\begin{abstract}
In modern engineering, physical processes are modelled using advanced computer simulation tools (e.g. finite element models) to assess and optimize their performance. Moreover, awareness is growing on concepts like structural reliability and robust design, hence making the efficient quantification and propagation of uncertainties a key challenge. A major part of those analyses is the characterization of uncertainty in the input, which is typically done with probabilistic variables. In the case of sparse data sets, however, probability theory is often not the optimal choice. Imprecise probabilities provide a more general framework accounting for both aleatory and epistemic uncertainties. The uncertainty propagation of imprecise probabilities leads to imprecise responses. In this context, an algorithm for solving imprecise structural reliability problems is presented. The algorithm transforms the imprecise problem into two precise structural reliability problems, which opens up possibilities for using traditional structural reliability analyses techniques. An adaptive experimental design algorithm based on Polynomial-Chaos-Kriging is used to efficiently estimate imprecise failure probabilities. The capabilities of the framework are illustrated through an application using an analytical function and a realistic engineering problem setting.
\end{abstract}

\section{INTRODUCTION}

In modern engineering, computational simulations, such as finite element models, have become a popular tool for predicting the behaviour of mechanical systems or engineering structures. The increasing knowledge in science leads to more and more complex computational models which require increasing computational power.

At the same time, the awareness on (quantitative) reliability, robustness and design optimization is growing (Rasmussen and Williams 2006). Engineers are more and more concerned with the quantification of uncertainties, which involves defining a computational model, determining a model for the uncertain input parameters, propagating the uncertainty and finally analysing the uncertainty in the quantity of interest (Sudret 2015).

After defining a proper computational model, the task is to characterize uncertainty in its input. It is common practice to model uncertain parameters with probability distributions, i.e. using concepts of probability theory. In many practical cases though, the knowledge and data to model such parameters are not sufficient. Thus, a more general framework is needed to capture both the natural variability (aleatory uncertainty) as well as the lack of knowledge (epistemic uncertainty). A wide variety of approaches have been proposed in the literature, such as Dempster-Shafer's theory (Dempster 1967, Shafer 1976), probabilityboxes (Ferson and Ginzburg 1996) and Bayesian hierarchical models (Gelman 2006). These frameworks are generally referred to as imprecise probabilities.

The propagation of imprecise probabilities through a computational model generally results in an imprecise quantity of interest. The analysis of this imprecise quantity requires different tools than those used in the context of a probabilistic input. Several algorithms have been developed for structural reliability analysis with probabilistic input, such as first-order reliability method (FORM), subset simulation, importance sampling and meta-modelling-based algorithms (Echard et al. 2011, Echard et al. 2013, Schöbi and Sudret 2014), which allow efficient (i.e. accurate and inexpensive) estimation of failure probabilities. However, it is not straightforward to apply these traditional algorithms in the context of imprecise structural reliability analysis (Alvarez et al. 2014, Zhang et al. 2015). In this paper, we elaborate a solution which combines imprecise structural reliability analysis with adaptive experimental design algorithms.

This paper is organized as follows. In a first step, the concept of imprecise probability is presented as a generalization of probability theory (Section 2). Then, the problem of imprecise structural reliability is discussed and a solution is proposed in Section 3. The 
proposed algorithm is illustrated in Section 4 in applications involving an analytical function as well as a realistic engineering problem.

\section{IMPRECISE PROBABILITY}

\subsection{Probability theory}

Traditionally, uncertainty in engineering has been treated with probability theory. Probability theory considers a probability space $(\Omega, \mathcal{F}, \mathbb{P})$, where $\Omega$ denotes an event space equipped with the $\sigma$-algebra $\mathcal{F}$ and a probability measure $\mathbb{P}$. In this context, random variables, denoted by capital letters, represent the mapping $X(\omega): \Omega \rightarrow \mathcal{D}_{X} \subset \mathbb{R}$, where $\omega \in \Omega$ is an elementary event. A realization of $X$ is denoted by the corresponding lower case letter $x$. When considering several random variables, we obtain a random vector $\boldsymbol{X}=\left[X_{1}, \ldots, X_{M}\right]$ and the corresponding realizations $\boldsymbol{x}=\left[x_{1}, \ldots, x_{M}\right]$.

Typically, a random variable $X$ is described by its cumulative distribution function (CDF) $F_{X}$, which describes the probability that $X<x$, i.e. $F_{X}(x)=\mathbb{P}(X<x)$. Any $\mathrm{CDF}$ has the following properties: it is monotonically non-decreasing, tends to zero for low values of $x$ and tends to one for large values of $x$.

As seen in the definition above, probability theory provides a single measure to describe the variability in $X$. In other words, it is assumed that the variability in $X$ is known and quantifiable through its CDF. Thus, variability is treated as the only source of uncertainty.

\subsection{Probability-boxes}

When the sources of uncertainty include natural variability (aleatory uncertainty) as well as lack of knowledge (epistemic uncertainty), a more general formulation is required to describe a random variable $X$. The basic idea of probability-boxes (p-boxes) is that the CDF cannot be given a precise formulation due to lack of knowledge. Hence, the uncertainty in $X$ is quantified by an upper- and a lower-bound CDF (Ferson and Ginzburg 1996, Ferson and Hajagos 2004), which are denoted by $\underline{F}_{X}$ and $\bar{F}_{X}$, respectively. For any value $x \in X$, the true but unknown CDF lies within these boundaries, i.e. $\underline{F}_{X}(x) \leq F_{X}(x) \leq \bar{F}_{X}(x), \forall x \in X$. The boundaries of the p-box $\left[\underline{F}_{X}, \bar{F}_{X}\right]$ mark the extreme cases of the real CDF and are thus themselves CDFs by definition.

An intermediate space is formed between these boundaries in the variable-CDF-graph which resembles a box (see Figure 1), hence the name probabilitybox. The particular case $\underline{F}_{X}(x)=\bar{F}_{X}(x), \forall x \in X$, corresponds to the probability-boxes degenerating into a single CDF. Then, conventional probability theory can be applied.

In the context of Dempster-Shafer's evidence theory (Shafer 1976) the boundary CDFs $\underline{F}_{X}$ and $\bar{F}_{X}$

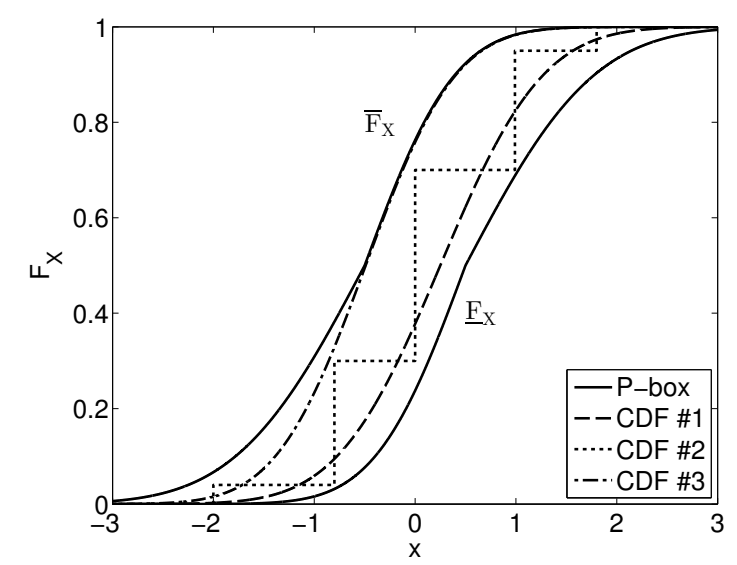

Figure 1: Free p-box - Sketch of $F_{X}=\left[\underline{F}_{X}, \bar{F}_{X}\right]$

can be interpreted as the belief and plausibility measures for the probability $\mathbb{P}(X \leq x)$. The belief describes the minimum amount of probability that must be associated to the event $X \leq x$, whereas the plausibility describes the maximum amount of probability that might be associated to the event $X \leq x$.

In the literature, two types of p-boxes are distinguished: free and parametric p-boxes. Free p-boxes are defined solely by $\left[\underline{F}_{X}, \bar{F}_{X}\right]$. This implies that the true $\mathrm{CDF} F_{X}$ can have an arbitrary shape as long as it follows the general properties of a CDF and it lies within the boundaries defined by $\underline{F}_{X}$ and $\bar{F}_{X}$. A variety of CDFs can be imagined of which Figure 1 illustrates a few realizations. In particular, it is possible to imagine a continuous function (CDF \#1 and \#3 in Figure 1) as well as CDFs of discrete random variables which allow only a set of values for $X$ (CDF \#2).

The second type of p-boxes are parametric p-boxes, which are defined by a family of distribution functions $F_{X}(x \mid \boldsymbol{\theta})$ whose parameters lie within an interval, i.e. $\theta_{i} \in\left[\underline{\theta}_{i}, \bar{\theta}_{i}\right]$. The definition of a parametric $\mathrm{p}$ box structure thus requires knowledge about the shape of the true CDF, which poses an additional constraint compared to the free p-box.

In this paper, we focus on the analysis of free $\mathrm{p}$ boxes as they describe a more general case of imprecise probabilities than parametric p-boxes.

\section{IMPRECISE STRUCTURAL RELIABILITY ANALYSIS}

\subsection{Computational model}

A computational model $\mathcal{M}$ is defined as a mapping of the $M$-dimensional input vector $\boldsymbol{x}$ to the output scalar $y$ :

$\mathcal{M}: \boldsymbol{x} \in \mathcal{D}_{\boldsymbol{X}} \subset \mathbb{R}^{M} \rightarrow y=\mathcal{M}(\boldsymbol{x}) \in \mathbb{R}$.

Due to uncertainties in the input vector $\boldsymbol{x}$, the latter is represented by an imprecise random vector $\boldsymbol{X}$ whose components $\left(X_{1}, \ldots, X_{M}\right)$ are assumed statistically independent throughout this paper for the sake of simplicity. Each component $X_{i}$ is modelled by a free p-box $\left[\underline{F}_{X_{i}}, \bar{F}_{X_{i}}\right]$. Hence, the joint 
probability-box $\left[\underline{F}_{\boldsymbol{X}}, \bar{F}_{\boldsymbol{X}}\right]$ can be obtained in terms of its marginal distributions, i.e. $\underline{F}_{X}=\prod_{i=1, \ldots, M} \underline{F}_{X_{i}}$ and $\bar{F}_{\boldsymbol{X}}=\prod_{i=1, \ldots, M} \bar{F}_{X_{i}}$. Finally, the imprecise random response $Y$ is obtained by propagating $\boldsymbol{X}$ through the computational model, i.e. $Y=\mathcal{M}(\boldsymbol{X})$. The corresponding free p-box of the response is denoted by $\left[\underline{F}_{Y}, \bar{F}_{Y}\right]$.

\subsection{Failure probability estimation}

In structural reliability analysis, the failure probability is defined as the probability that a response quantity of interest $Y$ is smaller than a given threshold value $y_{0}$ :

$P_{f}=\mathbb{P}\left(Y \leq y_{0}\right)=\mathbb{P}\left(\mathcal{M}(\boldsymbol{X}) \leq y_{0}\right)$.

Due to the fact that the input is modelled by p-boxes, different realizations of $F_{\boldsymbol{X}} \in\left[\underline{F}_{\boldsymbol{X}}, \bar{F}_{\boldsymbol{X}}\right]$ lead to different failure probabilities. Thus the imprecise failure probability consists of an interval $\left[\underline{P}_{f}, \bar{P}_{f}\right]$ rather than a single value. Figure 2 illustrates the p-box of $Y$ and the minimum and maximum failure probabilities. The minimum failure probability is obtained by $\underline{P}_{f}=\underline{F}_{Y}\left(y_{0}\right)$ whereas the maximum failure probability is obtained by $\bar{P}_{f}=\bar{F}_{Y}\left(y_{0}\right)$. This implies that the imprecise failure probability $\left[\underline{P}_{f}, \bar{P}_{f}\right]$ can be estimated when the boundary CDFs of the response $\mathrm{p}$ box are known. However, in a general case it is nontrivial to obtain $\left[\underline{F}_{Y}, \bar{F}_{Y}\right]$ efficiently.

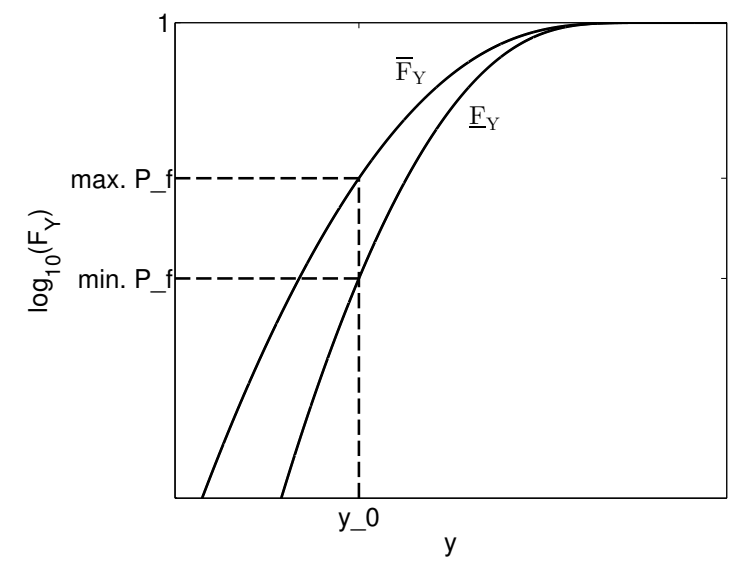

Figure 2: Response p-box and minimum/maximum failure probability

Note that for engineering applications, only the upper bound to the failure probability $\bar{P}_{f}$ is of interest (conservative estimate). Nevertheless the theory is developed for both bounds in the sequel.

\subsection{Problem conversion}

In order to be able to use standard reliability analysis tools available in the context of probability theory, we transform the imprecise reliability problem. Consider the random vector $C$ with length $M$ which is uniformly distributed in the unit-hypercube domain
$\mathcal{D}_{\boldsymbol{C}}=[0,1]^{M}$. Let the random variable $C_{i}$ describe the CDF value of $X_{i}$. Each $c_{i} \in[0,1]$ can be transformed into an interval in $\mathcal{D}_{X_{i}}$ through the inverse CDF of the p-box boundaries:

$\underline{x}_{i}\left(c_{i}\right)=\bar{F}_{X_{i}}^{-1}\left(c_{i}\right), \quad \bar{x}_{i}\left(c_{i}\right)=\underline{F}_{X_{i}}^{-1}\left(c_{i}\right)$.

Further, for each $c \in C$, we define a computational model for the minimum and maximum response in $[\underline{\boldsymbol{x}}(\boldsymbol{c}), \overline{\boldsymbol{x}}(\boldsymbol{c})]$ (Alvarez et al. 2014):

$\underline{\mathcal{M}}(\boldsymbol{c})=\min _{\boldsymbol{x} \in[\underline{\underline{x}}(\boldsymbol{c}), \overline{\boldsymbol{x}}(\boldsymbol{c})]} \mathcal{M}(\boldsymbol{x})$,

$\overline{\mathcal{M}}(\boldsymbol{c})=\max _{\boldsymbol{x} \in[\underline{\underline{\boldsymbol{x}}}(\boldsymbol{c}), \overline{\boldsymbol{x}}(\boldsymbol{c})]} \mathcal{M}(\boldsymbol{x})$.

We then define the probabilistic response variables for the two computational models as:

$\underline{Y}=\overline{\mathcal{M}}(\boldsymbol{C}), \quad \bar{Y}=\underline{\mathcal{M}}(\boldsymbol{C})$,

where $\underline{Y}$ has the $\mathrm{CDF} \underline{F}_{Y}$ and $\bar{Y}$ has the $\mathrm{CDF}$ $\bar{F}_{Y}$. Consequently, $\underline{P}_{f}=\mathbb{P}\left(\underline{Y} \leq y_{0}\right) \equiv \underline{F}_{Y}\left(y_{0}\right)$ and $\bar{P}_{f}=\mathbb{P}\left(\bar{Y} \leq y_{0}\right) \equiv \bar{F}_{Y}\left(y_{0}\right)$.

The definition of the two computational models $\underline{\mathcal{M}}$ and $\overline{\mathcal{M}}$ splits the estimation of the imprecise failure probability into two independent problems within the context of probability theory. The extreme failure probabilities are then defined as:

$\underline{P}_{f}=\mathbb{P}\left(\overline{\mathcal{M}}(\boldsymbol{C}) \leq y_{0}\right)$,

$\bar{P}_{f}=\mathbb{P}\left(\underline{\mathcal{M}}(\boldsymbol{C}) \leq y_{0}\right)$,

where the function argument $C$ is a uniform random variable over $[0,1]^{M}$. This implies that the failure probability can be estimated based on the probabilistic input model $\boldsymbol{C}$.

\subsection{Solution algorithms}

Eq. (7) and Eq. (8) define two structural reliability problems. Each of them can be solved by traditional structural reliability analyses, typically samplingbased methods, such as Monte Carlo simulation, importance sampling, subset simulation, and the firstorder-reliability method (FORM). For a specific problem, either of them may be preferable.

It should be noted that the evaluation of the computational models $\mathcal{M}$ and $\overline{\mathcal{M}}$ involves optimizations in the $M$-dimensional domain as seen in Eq. (4) and Eq. (5). Different optimization algorithms are available, amongst which are gradient-based algorithms, genetic algorithms and combinations of the two (socalled hybrid algorithms). The optimal choice for the optimization algorithm is problem-dependent.

The complexity of the computational model in Eq. (4) and Eq. (5) is higher than the one in Eq. (1) due to the required optimization. This makes the propagation of an $M$-dimensional input vector $c$ more expensive. One approach to reduce the computational 
costs is to use meta-models, which approximate the behaviour of the expensive-to-evaluate computational model with an inexpensive-to-evaluate function. A Kriging-based algorithm is discussed in the following.

\subsection{Imprecise-Adaptive-PC-Kriging}

The framework of Imprecise-Adaptive-PolynomialChaos-Kriging Monte Carlo simulation (Imprecise A-PCK-MCS) is summarized in Figure 3. It comprises two independent main blocks for $\mathcal{M}$ and $\overline{\mathcal{M}}$, which are marked by grey boxes. The algorithm for two blocks is identical except for the target computational model. For this reason, we use the notation $\underline{\overline{\mathcal{M}}}(\boldsymbol{C})$ in the following to represent both $\underline{\mathcal{M}}$ and $\overline{\mathcal{M}}$.

\subsubsection{Polynomial-Chaos-Kriging}

Kriging (a.k.a Gaussian process modelling) is a metamodelling technique which interprets the computational model as a realization of a Gaussian process (Santner et al. 2003):

$\overline{\bar{M}}(\boldsymbol{c}) \approx \mathcal{M}^{(\mathrm{K})}(\boldsymbol{c})=\boldsymbol{\beta}^{\top} \cdot \boldsymbol{f}(\boldsymbol{c})+\sigma^{2} Z(\boldsymbol{c}, \omega)$,

where $\boldsymbol{\beta}^{\top} \cdot \boldsymbol{f}(\boldsymbol{c})$ is the mean value of the Gaussian process (so-called trend), $\sigma^{2}$ is the process variance and $Z(\boldsymbol{c}, \omega)$ is a zero-mean, unit-variance stationary Gaussian process. The Gaussian process is characterized by an autocorrelation function $R=R\left(\left|\boldsymbol{c}-\boldsymbol{c}^{\prime}\right| ; \boldsymbol{\theta}\right)$ and its hyper-parameters $\boldsymbol{\theta}$.

A special case of Kriging is Polynomial-ChaosKriging (PC-Kriging) which uses a finite set of multivariate orthogonal polynomials as trend of a Kriging meta-model (Schöbi et al. 2015, Schöbi and Sudret 2014):

$\underline{\overline{\mathcal{M}}}(\boldsymbol{c}) \approx \mathcal{M}^{(\mathrm{PCK})}(\boldsymbol{c})=\sum_{\boldsymbol{\tau} \in \mathcal{T}} t_{\boldsymbol{\tau}} \psi_{\boldsymbol{\tau}}(\boldsymbol{c})+\sigma^{2} Z(\boldsymbol{c}, \omega)$,

where $\sum_{\boldsymbol{\tau} \in \mathcal{T}} t_{\boldsymbol{\tau}} \psi_{\boldsymbol{\tau}}(\boldsymbol{c})$ is a trend defined by a set of $|\mathcal{T}|$ multivariate orthogonal polynomials $\psi_{\boldsymbol{\tau}}(\boldsymbol{c})$ indexed by the multi-index $\boldsymbol{\tau}=\left\{\tau_{1}, \ldots, \tau_{M}\right\}$ and $t_{\boldsymbol{\tau}}$ are the corresponding coefficients. Due to the independence of the input variables, the multivariate polynomials can be constructed as tensor products of univariate polynomials:

$\psi_{\boldsymbol{\tau}}(\boldsymbol{c})=\prod_{i=1}^{M} \psi_{\tau_{i}}^{(i)}\left(c_{i}\right)$,

where $\psi_{\tau_{i}}^{(i)}\left(c_{i}\right)$ is a univariate polynomial of degree $\tau_{i}$ in the $i$-th variable. The orthogonality with respect to the input marginals is satisfied when:

$\left\langle\psi_{j}^{(i)}, \psi_{k}^{(i)}\right\rangle=\int_{\mathcal{D}_{i}} \psi_{j}^{(i)}(c) \psi_{k}^{(i)}(c) f_{C_{i}}(c) \mathrm{d} c=\delta_{j k}$, where $f_{C_{i}}$ is the marginal PDF of $C_{i}, \delta_{j k}=1$ for $j=$ $k$ and $\delta_{j k}=0$ otherwise. A compilation of common orthonormal univariate polynomials can be found in e.g. Sudret (2015).

Finally, the training of the PCKriging meta-model is achieved through a set of realizations of the input vector $\mathfrak{C}=\left\{\mathfrak{c}^{(1)}, \ldots, \mathfrak{c}^{(N)}\right\}$ and the corresponding responses $\mathcal{Y}=\left\{\mathcal{Y}^{(1)}=\underline{\overline{\mathcal{M}}}\left(\mathbf{c}^{(1)}\right), \ldots, \mathcal{Y}^{(N)}=\overline{\overline{\mathcal{M}}}\left(\mathfrak{c}^{(N)}\right)\right\} . \quad$ The optimal sparse set of polynomials $\mathcal{T}$ is obtained by least-angle regression as described in Schöbi et al. (2015). The Kriging parameters $\left\{\boldsymbol{t}, \sigma^{2}\right\}$ are estimated through the generalized least-squares solution for a given set of polynomials (Santner et al. 2003):

$\boldsymbol{t}(\boldsymbol{\theta})=\left(\mathbf{F}^{\top} \mathbf{R}^{-1} \mathbf{F}\right)^{-1} \mathbf{F} \mathbf{R}^{-1} \mathcal{Y}$,

$\sigma_{y}^{2}(\boldsymbol{\theta})=\frac{1}{N}(\mathcal{Y}-\mathbf{F} \boldsymbol{t})^{\top} \mathbf{R}^{-1}(\mathcal{Y}-\mathbf{F} \boldsymbol{t})$,

where $\mathbf{R}_{i j}=R\left(\left|\mathfrak{c}^{(i)}-\mathfrak{c}^{(j)}\right| ; \boldsymbol{\theta}\right)$ is the correlation matrix and $\mathbf{F}_{i j}=\psi_{j}\left(\mathbf{c}^{(i)}\right)$ is the information matrix. In cases where the correlation parameters $\boldsymbol{\theta}$ are unknown a-priori, their optimal value can be obtained through maximum-likelihood estimation.

The prediction of the response value $y$ of an arbitrary input sample $c$ is a Gaussian random variable characterized by its mean value and standard deviation (Santner et al. 2003):

$\mu_{\widehat{Y}}(\boldsymbol{c})=\boldsymbol{\psi}(\boldsymbol{c})^{\top} \boldsymbol{t}+\boldsymbol{r}(\boldsymbol{c})^{\top} \mathbf{R}^{-1}(\mathcal{Y}-\mathbf{F} \boldsymbol{t})$,

$$
\begin{aligned}
& \sigma_{\widehat{Y}}^{2}(\boldsymbol{c})= \\
& \sigma_{y}^{2}\left(1-\left\langle\boldsymbol{\psi}(\boldsymbol{c})^{\top} \boldsymbol{r}(\boldsymbol{c})^{\top}\right\rangle\left[\begin{array}{cc}
\mathbf{0} & \mathbf{F}^{\top} \\
\mathbf{F} & \mathbf{R}
\end{array}\right]^{-1}\left[\begin{array}{l}
\boldsymbol{\psi}(\boldsymbol{c}) \\
\boldsymbol{r}(\boldsymbol{c})
\end{array}\right]\right),
\end{aligned}
$$

where $\mu_{\widehat{Y}}(\boldsymbol{c})$ and $\sigma_{\widehat{Y}}^{2}(\boldsymbol{c})$ are the prediction mean value and variance, and $r_{i}(\boldsymbol{c})=R\left(\left|\boldsymbol{c}-\mathfrak{c}^{(i)}\right| ; \boldsymbol{\theta}\right)$ is the correlation between the new sample $c$ and the experimental design point $\mathfrak{c}^{(i)} \in \mathfrak{C}$.

\subsubsection{Adaptive experimental design}

The PC-Kriging model generally approximates the behaviour of $\overline{\mathcal{M}}$ most accurately close to the points of the experimental design $\mathfrak{C}$. However, these points are not always optimal for estimating the failure probability $P_{f}$. It is of interest to meta-model the region close to the limit state surface $\left(\overline{\mathcal{M}} \approx y_{0}\right)$, which is usually unknown a-priori. In this context, an adaptive experimental design algorithm, which iteratively adds samples to the experimental design in a guided way, can improve the accuracy of the estimation of $P_{f}$.

In this paper, we refer to the algorithms proposed by Echard et al. (2011) and applied to PC-Kriging in Schöbi and Sudret (2014) and Schöbi et al. (2015). The main steps are summarized here briefly: 


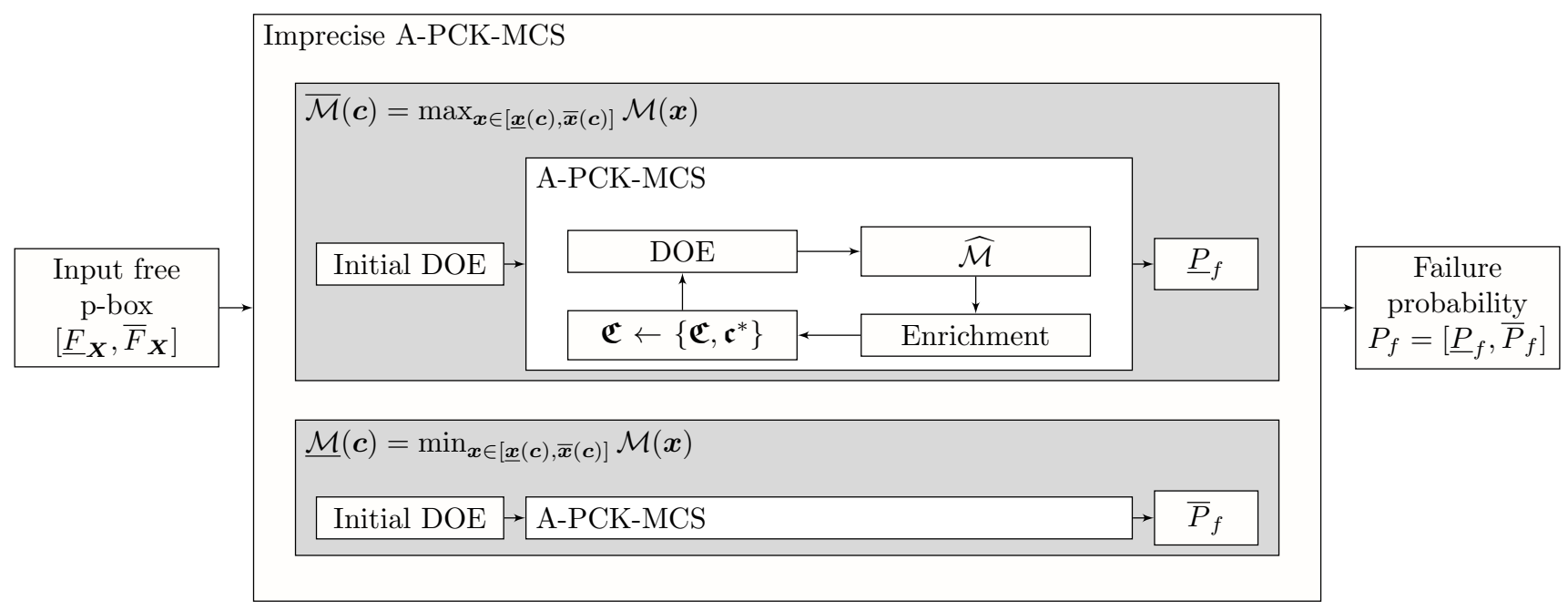

Figure 3: Imprecise A-PCK-MCS - Framework for obtaining the imprecise failure probability $\left[\underline{P}_{f}, \bar{P}_{f}\right]$.

1. Generate a small experimental design $\mathfrak{C}$ and compute the corresponding exact response $\mathcal{Y}$, where $\mathcal{Y}^{(i)}=\overline{\mathcal{M}}\left(\mathfrak{c}^{(i)}\right), i=1, \ldots, N$.

2. Train a meta-model $\mathcal{M}^{(\mathrm{PCK})}$ based on $\{\mathfrak{C}, \mathcal{Y}\}$.

3. Generate a large set of candidate samples $\mathcal{S}=\left\{\boldsymbol{c}_{1}, \ldots, \boldsymbol{c}_{n}\right\}$ from $\boldsymbol{C}$ and predict its response values with $\mathcal{M}^{(\mathrm{PCK})}$.

4. Estimate the failure probability:

$$
\widehat{P}_{f}^{0}=\mathbb{P}\left(\mu_{\widehat{Y}}(\boldsymbol{c}) \leq y_{0}\right) .
$$

5. Compute the probability of misclassification

$$
P_{m}\left(\boldsymbol{c}_{i}\right)=\Phi\left(\frac{\left|\mu_{\widehat{Y}}\left(\boldsymbol{c}_{i}\right)-y_{0}\right|}{\sigma_{\widehat{Y}}\left(\boldsymbol{c}_{i}\right)}\right),
$$

for each $\boldsymbol{c}_{i} \in \mathcal{S}$, where $\Phi(\cdot)$ is the CDF of the standard Gaussian distribution $(\mu=0, \sigma=1)$. Select the next best sample to be added to the experimental design as $\mathfrak{c}^{*}=\underset{i}{\arg \max } P_{m}\left(\boldsymbol{c}_{i}\right)$.

6. Add the selected sample $\mathfrak{c}^{*}$ to the experimental design, $\mathfrak{C} \leftarrow\left\{\mathfrak{C}, \mathfrak{c}^{*}\right\}$, compute the corresponding response $\mathcal{Y}^{*}=\underline{\overline{\mathcal{M}}}\left(\mathfrak{c}^{*}\right)$ and $\mathcal{Y} \leftarrow\left\{\mathcal{Y}, \mathcal{Y}^{*}\right\}$.

7. Go back to step 2 to train the meta-model with the enhanced experimental design unless the following convergence criterion is fulfilled:

$$
\frac{\widehat{P}_{f}^{+}-\widehat{P}_{f}^{-}}{\widehat{P}_{f}^{0}} \leq 5 \%,
$$

where $\widehat{P}_{f}^{ \pm} \stackrel{\text { def }}{=} \mathbb{P}\left(\mu_{\widehat{Y}}(\boldsymbol{c}) \mp 2 \sigma_{\widehat{Y}}(\boldsymbol{c}) \leq y_{0}\right)$.

The estimate of the failure probability is finally obtained with the last meta-model $\mathcal{M}^{\text {(PCK) }}$. For further details, the reader is referred to Schöbi and Sudret (2014) and Schöbi et al. (2015). Note that the entire adaptive experimental design algorithm is conducted twice, for $\underline{\mathcal{M}}$ and $\overline{\mathcal{M}}$.

\subsubsection{Meta-model domain}

The use of domain $\mathcal{D}_{C_{i}}$ for meta-modelling is suitable in cases where the p-box for variable $X_{i}$ is bounded. Then, the isoprobabilistic transform from $\mathcal{D}_{C_{i}}$ to $\mathcal{D}_{X_{i}}$ tends to be only slightly non-linear. When the p-box in $X_{i}$ is unbounded, however, the transform (Eq. (3)) is highly non-linear and may lead to convergence issues in the meta-modelling process. The explanation lies in the shape of the failure domain. When $X_{i}$ is unbounded and the failure probability is small, the size of the failure domain $\mathcal{D}_{f, C} \subset \mathcal{D}_{C}$ is proportional to the failure probability, i.e. $\mathcal{D}_{f, C} \ll 1$. In contrast, in the domain $\mathcal{D}_{\boldsymbol{X}}$, the corresponding failure domain $\mathcal{D}_{f, \boldsymbol{X}}$ is usually not compact and the corresponding limit state surface $\left(\underline{\overline{\mathcal{M}}}=y_{0}\right)$ can be meta-modelled more efficiently.

Thus, in the case of unbounded input p-boxes in $X_{i}$, we propose to apply imprecise A-PCK-MCS on an auxiliary space $\mathcal{D}_{\tilde{X}_{i}}$ and the corresponding auxiliary variables $\tilde{X}_{i}$, which is characterized by the following so-called mean $C D F$ :

$\tilde{F}_{X_{i}}\left(x_{i}\right)=\frac{\underline{F}_{X_{i}}\left(x_{i}\right)+\bar{F}_{X_{i}}\left(x_{i}\right)}{2}$.

$\tilde{F}_{X_{i}}$ describes an average CDF curve of all possible CDFs of the input free p-box in $X_{i}$. $\tilde{X}_{i}$ can then be transformed into $C_{i}$ by the isoprobabilistic transform $C_{i}=\tilde{F}_{X_{i}}\left(X_{i}\right)$. Consider the index sets of bounded variables $\mathcal{B}^{(b)}$ and of unbounded variables $\mathcal{B}^{(u)}$, such that $\mathcal{B}^{(b)} \cap \mathcal{B}^{(u)}=\emptyset$ and $\left\{\mathcal{B}^{(b)} \cup \mathcal{B}^{(u)}\right\}=\{i, i=1, \ldots, M\}$. Then, a new input vector $\boldsymbol{C}^{(\tilde{X})}$ is defined:

$C_{i}^{(\tilde{X})}=\left\{\begin{array}{ll}C_{i} & \text { if } i \in \mathcal{B}^{(b)} \\ \tilde{X}_{i} & \text { if } i \in \mathcal{B}^{(u)}\end{array}\right.$.

The corresponding computational model can then be written as a function of $\underline{\overline{\mathcal{M}}}$ as:

$\underline{\overline{\mathcal{M}}}^{(\tilde{X})}\left(\boldsymbol{C}^{(\tilde{X})}\right) \equiv \underline{\overline{\mathcal{M}}}(\boldsymbol{C})$. 
The adaptive experimental design algorithm can be implemented for $\overline{\mathcal{M}}^{(\tilde{X})}$, which promises a better convergence than $\overline{\mathcal{M}}$, due to the separate treatment of the unbounded variables.

\section{APPLICATION}

\subsection{Analytical function}

We define the following analytical model of varying dimension $M$ :

$g_{1}(\boldsymbol{x})=\sum_{i=1}^{M} x_{i}$,

where the components $x_{i} \in \boldsymbol{x}$ are modelled by independent free p-boxes. The boundary CDFs are modelled by Gaussian distributions:

$$
\begin{aligned}
& \underline{F}_{X_{i}}\left(x_{i}\right)=F_{\mathcal{N}}\left(x_{i} \mid \bar{\mu}=1.5, \sigma=1\right) . \\
& \bar{F}_{X_{i}}\left(x_{i}\right)=F_{\mathcal{N}}\left(x_{i} \mid \underline{\mu}=0.5, \sigma=1\right),
\end{aligned}
$$

where $F_{\mathcal{N}}\left(x_{i} \mid \mu, \sigma\right)$ describes the CDF of a Gaussian distribution with mean value $\mu$ and standard deviation $\sigma$.

Failure is defined as $g_{1} \leq 0$ and the corresponding failure probability reads $P_{f}=\mathbb{P}\left(g_{1}(\boldsymbol{X}) \leq 0\right)$. Due to the monotonicity of $g_{1}$ and the shape of $\left[\underline{F}_{X_{i}}, \bar{F}_{X_{i}}\right]$, the extreme failure probabilities can be estimated by:

$$
\begin{aligned}
& \underline{P}_{f}(M)=\Phi\left(\frac{-M \cdot \bar{\mu}}{\sqrt{M \cdot \sigma^{2}}}\right)=\Phi(-\sqrt{M} \cdot \bar{\mu}), \\
& \bar{P}_{f}(M)=\Phi\left(\frac{-M \cdot \underline{\mu}}{\sqrt{M \cdot \sigma^{2}}}\right)=\Phi(-\sqrt{M} \cdot \underline{\mu}) .
\end{aligned}
$$

The values $\bar{\mu}=1.5, \mu=0.5$ are used in the numerical experiments. The Imprecise A-PCK-MCS algorithm is initialized with an initial experimental design of $N_{0}=5 \cdot M$ Latin-hypercube samples and a Monte Carlo population $\mathcal{S}$ of $n=10^{6}$ samples.

The results are summarized in Table 1 and Table 2, which list the estimates of the failure probability $\widehat{P}_{f}$ and the number of model runs, i.e. the initial size of the experimental design $N_{0}$ and the number of added samples $N_{1}$. We denote $N_{\text {tot }}=N_{0}+N_{1}$.

The results show that the imprecise failure probability $\left[\underline{P}_{f}, \bar{P}_{f}\right]$ is estimated accurately for the presented dimensions $M$. For growing dimensionality $M$, the total number of model runs increases. Overall though, the results are obtained with a small number of runs of the computational model $\overline{\mathcal{M}}$. For all cases, the adaptive algorithm is stopped according to the criterion in Section 3.5.2. An exception is the case $\left\{M=5, \mathcal{D}_{C}\right\}$, where the estimate of the failure probability is stable after $N_{1}=200$ iterations but the convergence criterion is not fulfilled.
The difference in the meta-model domains $\mathcal{D}_{C}$ and $\mathcal{D}_{\tilde{\boldsymbol{X}}}$ (and the corresponding $\overline{\mathcal{M}}$ and $\overline{\mathcal{M}}^{(\tilde{X})}$ ) is visible in the comparison of Table 1 and Table 2. For $M=2$ and $M=5$, meta-modelling in $\mathcal{D}_{\tilde{\boldsymbol{X}}}$ leads to faster convergence of the estimate of the failure probability as seen by the lower total number of model evaluations $N_{t o t}$. This is due to the fact that the computational model $\mathcal{M}$ behaves linearly in $\boldsymbol{X}$ and thus also linearly in $\tilde{\boldsymbol{X}}$. PC-Kriging is capable of capturing the linear trend easily and thus predicting accurately any other $\tilde{\boldsymbol{x}} \in \tilde{\boldsymbol{X}}$.

Table 1: Analytical function - summary of the imprecise reliability analysis using imprecise A-PCK-MCS in the $\mathcal{D}_{C}$ domain.

\begin{tabular}{llll}
\hline & & $\underline{P}_{f}$ & $\bar{P}_{f}$ \\
\hline$M=2$ & $P_{f}$ & $1.69 \cdot 10^{-2}$ & $2.40 \cdot 10^{-1}$ \\
& $\widehat{P}_{f}$ & $1.70 \cdot 10^{-2}$ & $2.40 \cdot 10^{-1}$ \\
& $N_{t o t}$ & $10+127=137$ & $10+105=115$ \\
\hline$M=5$ & $P_{f}$ & $3.98 \cdot 10^{-4}$ & $1.32 \cdot 10^{-1}$ \\
& $\widehat{P}_{f}$ & $3.91 \cdot 10^{-4}$ & $1.31 \cdot 10^{-1}$ \\
& $N_{\text {tot }}$ & $>225$ & $>225$ \\
\hline
\end{tabular}

Table 2: Analytical function - summary of the imprecise reliability analysis using imprecise A-PCK-MCS in the $\mathcal{D}_{\tilde{X}}$ domain

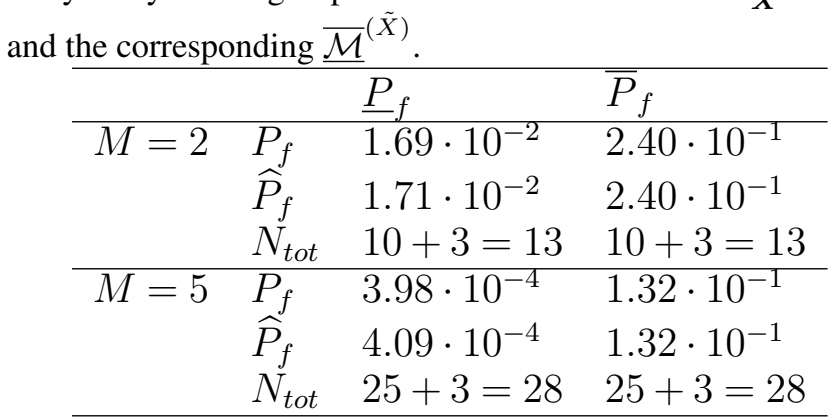

In order to visualize the behaviour of the adaptive algorithm, the experimental design of the meta-model is analysed. Figure 4 shows distinct experimental designs for the case of $M=2$. The blue rectangles represent the optimization domains of the initial experimental design samples in the input domain $\mathcal{D}_{\boldsymbol{X}}$, i.e. $\left[\underline{\boldsymbol{x}}\left(\mathfrak{c}^{(i)}\right), \overline{\boldsymbol{x}}\left(\mathfrak{c}^{(i)}\right)\right]$ for $i=1, \ldots, N_{0}$. The red and green rectangles represent the corresponding domains of the first $N_{1}=50$ added samples for $\overline{\mathcal{M}}$ and $\underline{\mathcal{M}}$ respectively. The black line marks the limit state surface $g_{1}(\boldsymbol{x})=0$. Figure 4 visualizes nicely the behaviour of the Imprecise A-PCK-MCS algorithm as the added samples approach the limit state surface from the left and right side for $\overline{\mathcal{M}}$ and $\mathcal{M}$, respectively. This implies that the meta-model $\overline{\overline{\mathcal{M}}}$ models accurately the limit state surface with each added sample (i.e. rectangle).

\subsection{Simply supported truss}

Consider the simply supported, linear-elastic truss presented in Hurtado (2013) and sketched in Figure 5. The computational model $\mathcal{M}$ consists of 


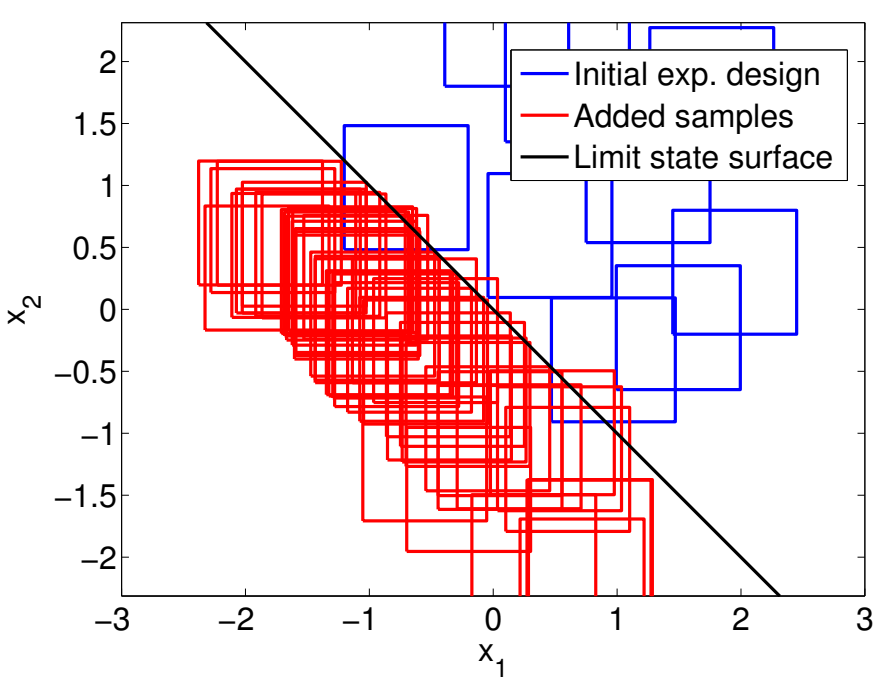

(a) $\overline{\mathcal{M}}$ for estimating $\underline{P}_{f}$

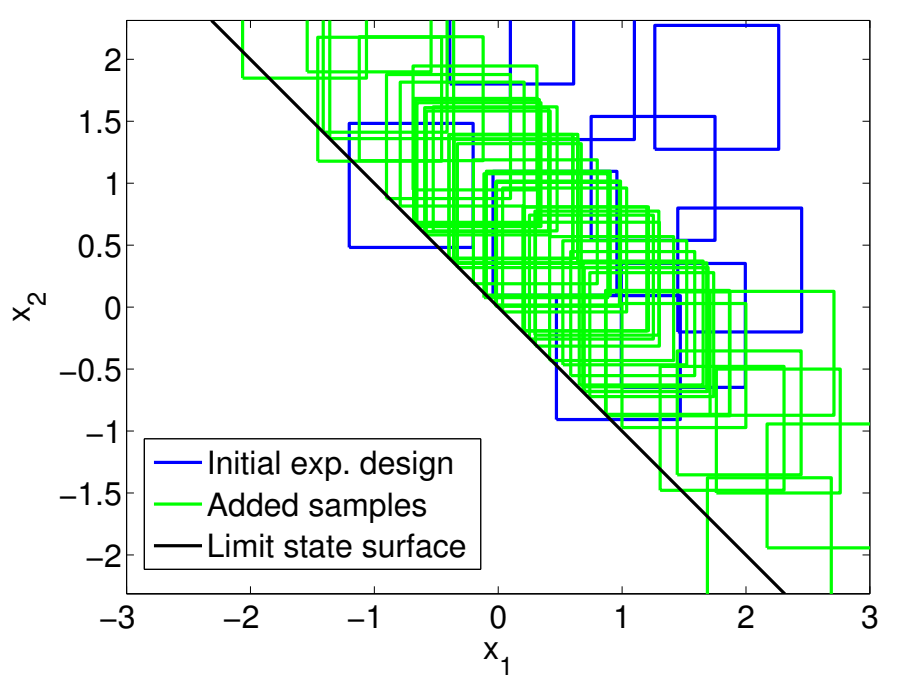

(b) $\underline{\mathcal{M}}$ for estimating $\bar{P}_{f}$

Figure 4: Analytical function - experimental design in $\mathcal{D}_{\boldsymbol{X}}$. (The initial samples are blue, the added samples for $\overline{\mathcal{M}}$ are red and the added samples for $\underline{\mathcal{M}}$ are green)

a finite element model of this truss structure. All bars are modelled with a constant Young's modulus $E=200 \cdot 10^{9} \mathrm{~Pa}$. The cross sectional area of the bars varies: $A=0.00535 \mathrm{~m}^{2}$ for the bars marked by $\bullet, A=0.0068 \mathrm{~m}^{2}$ for the bars marked by $\circ$ and $A=0.004 \mathrm{~m}^{2}$ for the remaining bars. The uncertainty in the seven independent loads $P_{i}$ is modelled by free p-boxes whose boundary CDFs are:

$\underline{F}_{P_{i}}\left(p_{i}\right)=\min \left[\begin{array}{l}F_{\mathcal{L N}}\left(p_{i} \mid \mu=105 \mathrm{kN}, \sigma=13 \mathrm{kN}\right) \\ F_{\mathcal{L N}}\left(p_{i} \mid \mu=105 \mathrm{kN}, \sigma=17 \mathrm{kN}\right)\end{array}\right]$,

$\bar{F}_{P_{i}}\left(p_{i}\right)=\max \left[\begin{array}{l}F_{\mathcal{L N}}\left(p_{i} \mid \mu=95 \mathrm{kN}, \sigma=13 \mathrm{kN}\right) \\ F_{\mathcal{L N}}\left(p_{i} \mid \mu=95 \mathrm{kN}, \sigma=17 \mathrm{kN}\right)\end{array}\right]$,

where $F_{\mathcal{L N}}\left(p_{i} \mid \mu, \sigma\right)$ describes the CDF of a lognormal distribution with mean value $\mu$ and standard deviation $\sigma$. Note that these bounds are identical to those of a parametric p-box with lognormal distributions, in which parameters $\mu$ and $\sigma$ vary in ranges $\mu \in[95,105] \mathrm{kN}$ and $\sigma \in[13,17] \mathrm{kN}$. This parametric p-box was used in Hurtado (2013) whereas we consider a free p-box here. The resulting free p-box is illustrated in Figure 6. The quantity of interest is the deflection at midspan, denoted by $u_{4}$ (see Figure 5), as a function of the seven imprecise loads. In the context of structural reliability analysis, we define failure as $u_{4} \geq 0.029 \mathrm{~m}$ and the corresponding failure probability $P_{f}=\mathbb{P}\left(0.029 \mathrm{~m}-u_{4}(\boldsymbol{P}) \leq 0\right)$.

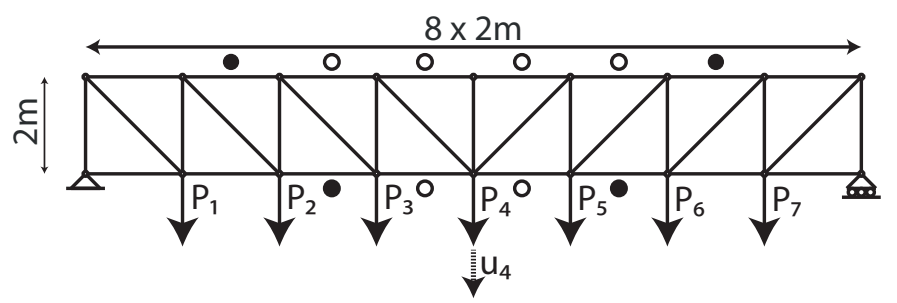

Figure 5: Truss structure - sketch of the geometry including the seven imprecise loads $P_{i}$ and the target deflection $u_{4}$

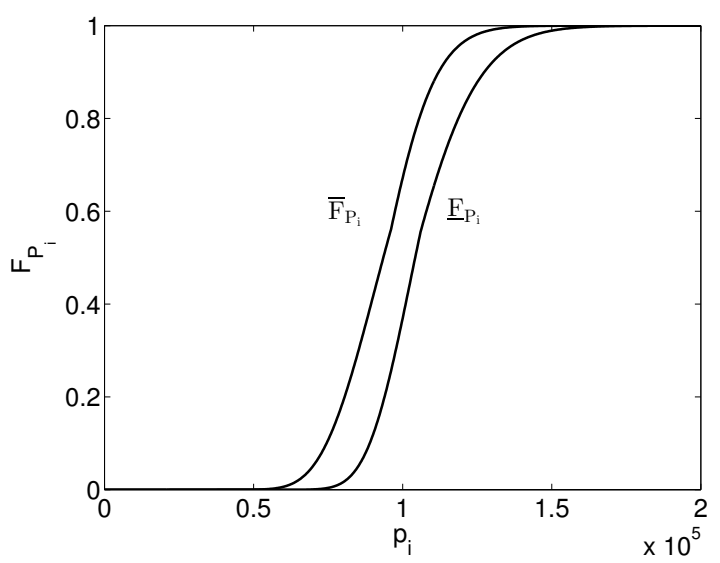

Figure 6: Truss structure - input free p-box for each of the seven imprecise loads $P_{i}$

The imprecise A-PCK-MCS algorithm is initialized with an experimental design of $N_{0}=10$ Latinhypercube samples and a Monte Carlo population $\mathcal{S}$ of $n=10^{6}$ samples. Due to the assumption that the failure probabilities are small, the meta-model for $\overline{\mathcal{M}}^{(\tilde{X})}$ is trained in the auxiliary space $\mathcal{D}_{\tilde{X}}$ (see Eq. (17)) rather than in the space $\mathcal{D}_{C}$. This ensures a more efficient estimation of the failure probabilities because the input variables are unbounded and the failure domain (where $\mathcal{M}^{(\mathrm{PCK})} \leq 0$ ) in $\mathcal{D}_{\tilde{\boldsymbol{X}}}$ is larger than in $\mathcal{D}_{C}$ (see also Section 3.5.3).

The results are summarized in Table 3 , in which the estimates of the failure probability are compared for a preset $N_{t o t}=110$. The estimates of the failure probability are accurate despite the small number of computational model runs. This illustrates the efficiency of the proposed approach. In particular, it is possible to estimate the bounds of the failure probability with a total of 220 runs of $\underline{\overline{\mathcal{M}}}^{(\tilde{X})}$. 
Table 3: Truss structures - summary of the imprecise reliability analysis using imprecise A-PCK-MCS

\begin{tabular}{llll}
\hline & & $\underline{P}_{f}$ & $\bar{P}_{f}$ \\
\hline MC & $\widehat{P}_{f}$ & $2.10 \cdot 10^{-4}$ & $9.20 \cdot 10^{-2}$ \\
& $n$ & $10^{6}$ & $10^{6}$ \\
\hline \multirow{2}{*}{ APCK } & $\widehat{P}_{f}$ & $2.25 \cdot 10^{-4}$ & $9.21 \cdot 10^{-2}$ \\
& $N_{\text {tot }}$ & $10+100=110$ & $10+100=110$ \\
\hline
\end{tabular}

It is interesting to note that the imprecision in the description of the seven loads leads to a significant uncertainty in the failure probability. A variation of $10 \%$ in the mean value and $20 \%$ in the standard deviation of the distribution function of $P_{i}$ leads to a factor $\bar{P}_{f} / \underline{P}_{f} \approx 200$ in the estimate of the failure probability. The failure probability is highly sensitive to the definition of the input parameters due to its large dependency on the tail of the input distributions.

\section{CONCLUSIONS}

This paper deals with the propagation of uncertainty in the input of a computational model. In engineering practice a typical scenario is the case where data generation is costly and thus limited. Due to the sparsity of proper calibration data, the input parameters are modelled as imprecise probabilities accounting for both aleatory and epistemic uncertainties. One way to capture this lack of knowledge is to use probabilityboxes which provide an upper and lower bound for the cumulative distribution function of variables.

In the context of imprecise probabilities, the estimation of bounds on the failure probability is not straightforward. In this paper, we propose a transformation of the imprecise structural reliability problem into two traditional structural reliability problems. We define two computational models as functions of an auxiliary variable set, so that the lower and upper boundary of the imprecise failure probability can be estimated independently.

The transformation allows one to use advanced structural reliability methods, such as the AdaptivePC-Kriging algorithm which is adopted in this paper. Adaptive-PC-Kriging is an adaptive experimental design algorithm which efficiently (accurately and inexpensively) estimates failure probabilities.

The capabilities of the proposed algorithm are illustrated by an analytical function and a realistic engineering problem. In both cases, the proposed algorithm is capable of estimating the bounds to the failure probability accurately with only a small number of runs of the exact computational model. This is of major significance in practice where computational resources are typically limited.

Further studies will include modifications of the proposed algorithm to accurately estimate rare event probabilities for which crude Monte Carlo simulation is not efficient.

\section{REFERENCES}

Alvarez, D. A., J. E. Hurtado, \& F. Uribe (2014). Estimation of the lower and upper probabilities of failure using random sets and subset simulation. In M. Beer, S.-K. Au, and J. W. Hall (Eds.), Vulnerability, Uncertain. Risk, Liverpool, UK, pp. 905-914. ASCE.

Dempster, A. P. (1967). Upper and lower probabilities induced by multivalued mapping. Ann. Math. Stat. 38(2), 325-339.

Echard, B., N. Gayton, \& M. Lemaire (2011). AK-MCS: an active learning reliability method combining Kriging and Monte Carlo simulation. Structural Safety 33(2), 145-154.

Echard, B., N. Gayton, M. Lemaire, \& N. Relun (2013). A combined Importance Sampling and Kriging reliability method for small failure probabilities with time-demanding numerical models. Reliab. Eng. Syst. Saf. 111, 232-240.

Ferson, S. \& L. R. Ginzburg (1996). Different methods are needed to propagate ignorance and variability. Reliab. Eng. Sys. Safety 54(2-3), 133-144.

Ferson, S. \& J. G. Hajagos (2004). Arithmetic with uncertain numbers: rigorous and (often) best possible answers. Reliab. Eng. Sys. Safety 85(1-3), 135-152.

Gelman, A. (2006). Prior distributions for variance parameters in hierarchical models (comment on article by Browne and Draper). Bayesian Anal. 1(3), 515-534.

Hurtado, J. E. (2013). Assessment of reliability intervals under input distributions with uncertain parameters. Prob. Eng. Mech. 32, 80-92.

Rasmussen, C. E. \& C. K. I. Williams (2006). Gaussian processes for machine learning (Internet ed.). Adaptive computation and machine learning. Cambridge, Massachusetts: MIT Press.

Santner, T. J., B. J. Williams, \& W. I. Notz (2003). The Design and Analysis of Computer Experiments. Springer, New York.

Schöbi, R. \& B. Sudret (2014). Combining polynomial chaos expansions and Kriging for solving structural reliability problems. In P. Spanos and G. Deodatis (Eds.), Proc. 7th Int. Conf. on Comp. Stoch. Mech (CSM7), Santorini, Greece.

Schöbi, R. \& B. Sudret (2014). PC-Kriging: a new metamodelling method combining Polynomial Chaos Expansions and Kriging. In Proc. 2nd Int. Symp. on Uncertainty Quantification and Stochastic Modelling, Rouen, France.

Schöbi, R., B. Sudret, \& S. Marelli (2015). Rare event estimation using Polynomial-Chaos-Kriging. ASCE-ASME J. Risk Uncertainty Eng. Syst., Part A: Civ. Eng, submitted.

Schöbi, R., B. Sudret, \& J. Wiart (2015). Polynomial-chaosbased Kriging. Int. J. Uncertainty Quantification 5(2), 171193.

Shafer, G. (1976). A mathematical theory of evidence. Princeton, NJ: Princeton University Press.

Sudret, B. (2015). Polynomials chaos expansions and stochastic finite element methods. In K.-K. Phoon and J. Ching (Eds.), Risk Reliab. Geotech. Eng., Chapter 5. Taylor and Francis.

Zhang, Z., C. Jiang, G. Wang, \& X. Han (2015). First and second order approximate reliability analysis methods using evidence theory. Reliab. Eng. Syst. Safety 137, 40-49. 